\title{
New: ADO Communications
}

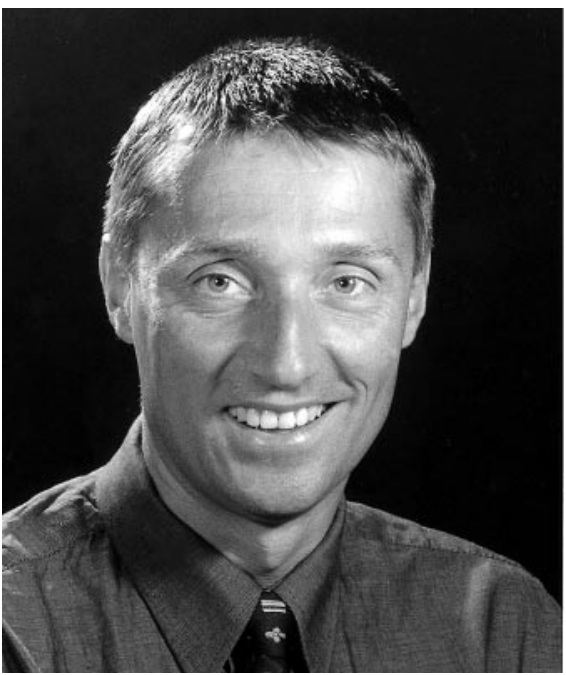

Die „Aktuelle Dermatologie“ ist seit vielen Jahren das Publikationsorgan der Arbeitsgemeinschaft Dermatologische Onkologie (ADO), die sowohl in der Deutschen Krebsgesellschaft als auch in der Deutschen Dermatologischen Gesellschaft beheimatet ist. Die ADO wird den dermato-onkologisch Interessierten unter Ihnen seit den 90erJahren bekannt sein als eine schlagkräftige, jung dynamische Organisation, die sich insbesondere der Therapieoptimierung bei Patienten mit Hauttumoren angenommen hat. Im Jahr 2004 möchten wir den Lesern in den monatlichen Ausgaben der „Aktuellen Dermatologie“ das Spektrum der ADO-Aktivitäten sowie Neuigkeiten und auch ein wenig Berufspolitik darstellen. Sie finden die Mitteilungsseite der ADO jeweils vor dem Kongresskalender im Heft.

In den acht Jahren seit der Initiierung der ersten ADO-Studie unter der Leitung von Herrn Professor Tilgen (Homburg/Saar) wurden mehr als 2800 Patienten in mittlerweile 13 Therapieoptimierungsprotokolle eingebracht. Damit gehört die ADO auch international zu den inzwischen etablierten großen Organisationen. 330 Mitglie- der und 91 überwiegend dermatologische Zentren, die sich an den bisherigen Protokollen beteiligt haben, zeugen von einem breiten Interesse an den vielfältigen Tätigkeiten der Organisation.

Darüber hinaus hat die ADO in den letzten Jahren zahlreiche Fortbildungsveranstaltungen zur praktischen Dermato-Onkologie, geeignet für die Klinik und für die Praxis, veranstaltet. Seit dem Jahr 2003 wird das Fortbildungsprogramm der ADO abgerundet durch Zertifizierungskurse für klinische Studien, die heute in zunehmendem Maß gefordert werden. Ein wesentlicher Verdienst kommt der ADO auch bei der Entwicklung von Leitlinien zur Diagnostik und Therapie von Hauttumoren zu. Hier ist im Jahre 2004 gleich zu Beginn mit neuen Therapieleitlinien zu rechnen, die auch in der „Aktuellen Dermatologie“ veröffentlicht werden sollen.

Neben den Studienaktivitäten und den Leitlinien soll auch das Thema der Dermato-Onkologie in der Praxis („onkologisch versierter Arzt“) in einem der nächsten Beiträge näher beleuchtet werden. Außerdem möchten wir Ihnen auch die „Deutsche Hautkrebsstiftung“ in Frankfurt ausführlich vorstellen.

Gegenstand des ersten Beitrages der ADO soll der neu gestaltete Internetauftritt sein, der sowohl Laien als auch Experten Zugang zu Informationen jeglicher Facette zu Hauttumoren geben soll. Unter der Aegide von C. Papavassilis (Gießen) wurde innerhalb von nur kurzer Zeit eine Internetplattform erstellt, bei der momentan täglich 200 Zugriffe erfolgen.

Der Dank der ADO gehört Frau Rettenmaier vom Thieme Verlag, die durch den steten Kontakt zu unserer Organisation die monatlichen Beiträge der ADO angeregt hat.

Mit freundlichen Grüßen im Namen des gesamten ADO-Vorstandes

Ihr A. Hauschild 